\title{
ArcheoSciences
}

Revue d'archéométrie

\section{Teneurs totales en plomb en fonction de la profondeur dans les sols «naturels »}

\author{
Le point de vue d'un pédologue
}

Total lead concentrations as a function of depth in "natural" soils A pedologist's viewpoint

\section{Denis Baize}

\section{OpenEdition}

\section{Journals}

\section{Édition électronique}

URL : http://journals.openedition.org/archeosciences/2709

DOI : 10.4000/archeosciences.2709

ISSN : 2104-3728

\section{Éditeur}

Presses universitaires de Rennes

\section{Édition imprimée}

Date de publication : 10 avril 2010

Pagination : 127-135

ISBN : 978-2-7535-1407-2

ISSN : $1960-1360$

\section{Référence électronique}

Denis Baize, «Teneurs totales en plomb en fonction de la profondeur dans les sols «naturels » », ArcheoSciences [En ligne], 34 | 2010, mis en ligne le 11 avril 2013, consulté le 19 janvier 2021. URL: http://journals.openedition.org/archeosciences/2709; DOI : https://doi.org/10.4000/archeosciences. 2709 


\title{
Teneurs totales en plomb en fonction de la profondeur dans les sols « naturels" Le point de vue d'un pédologue
}

\author{
Total lead concentrations as a function of depth in "natural" soils \\ A pedologist's viewpoint
}

\author{
Denis BAIZE*
}

\begin{abstract}
Résumé : Ce petit texte rappelle un certain nombre de généralités relatives aux éléments en traces (dont le plomb) dans les « sols naturels ", c'està-dire les sols agricoles ou forestiers non recouverts par des matériaux archéologiques et dont la morphologie, héritée de l'action des processus pédogénétiques, n’a pas été complètement effacée par des remaniements anthropiques.

Sont présentés brièvement et illustrés : la définition des " concentrations pédogéochimiques naturelles » et des " teneurs agricoles habituelles »; des données statistiques françaises portant sur plus de 10000 horizons de surface labourés et sur 260 solums analysés en région Nord-Pas de Calais; la faible mobilité du plomb dans les sols (à l'exception du cas des Podzosols); les flux de retombées atmosphériques et leur variabilité tant spatiale que temporelle; deux techniques pour déceler les contaminations anthropiques et évaluer leurs niveaux (relative topsoil enhancement et " comparaisons verticales").
\end{abstract}

\begin{abstract}
This paper presents some general points relative to trace metals (especially lead) in "natural " soils. These are agricultural or forested soils which have not been buried by archaeological material and the morphology of which, inherited from the action of soil forming processes, has not been totally erased by anthropogenic reworking.

Are briefly mentioned and illustrated: the definition of "natural pedogeochemical concentrations" and "usual agricultural contents"; statistical data for more than 10000 ploughed surface horizons nation-wide and 260 soil pits studied in the Nord-Pas de Calais region; the low mobility of lead in soils (with the noticeable exception of Podzosols); the fluxes of atmospheric fallout and their high variability in space and time; two techniques used in order to detect anthropogenic contaminations and assess their levels: relative topsoil enhancement (RTE) and "vertical comparison".

The RTE is the mere ratio of the total lead concentration in the surface layer $(0-15 \mathrm{~cm})$ divided by the concentration found at a depth of $30-45 \mathrm{~cm}$. A value $>4$ may indicate strong contamination by human activities. Unfortunately, this ratio is highly depending on the pedological nature of the semideep horizon and must be cautiously handled. It seems better to resort to the "vertical comparison" which is a simple and inexpensive approach.

The latter consists in interpreting the distribution of total lead contents with depth, for 4-5 or 6 superposed horizons. A high lead content in the topsoil contrasting with low values at depth is rather an evidence of pollution. On the contrary, a high concentration in the topsoil keeping steady or going increasing with depth is rather the indication of a natural geochemical anomaly.
\end{abstract}

Mots clé : plomb, contamination, sols, pédologie.

Key words: lead, contamination, soils, pedology.

* INRA, UR 0272 Science du Sol, Centre d'Orléans - CS 40001 Ardon, 45075 Orléans Cedex 2. 
Pour le pédologue, les sols (ou couvertures pédologiques) sont des formations naturelles continues, tridimensionnelles, situées à la surface de la croûte terrestre et formées par altération de matériaux géologiques. Ce que nous appelons sols "naturels" sont ceux formés initialement par les processus naturels de pédogenèse et plus ou moins modifiés ensuite par les activités humaines (notamment déboisements, agricultures, foresterie, etc.). Dans notre esprit, "sols naturels " s'oppose aux sols des archéologues (sols d'ateliers, sols de grottes, sols urbains).

\section{GÉNÉralités Relatives AU PLOMB}

Les géochimistes ont défini le plomb comme un élément en trace métallique (ETM). En effet, dans la croûte terrestre continentale, il n'est présent qu'en traces (en moyenne $16 \mathrm{mg} / \mathrm{kg}$ ). Pour les ETM, on compte en $\mathrm{mg} / \mathrm{kg}$ (ou ppm), à la différence des «éléments majeurs " comme le fer, l’aluminium ou le magnésium pour lesquels les concentrations sont exprimées en pour cent ou en pour mille.

Pour le plomb comme pour d'autres métaux potentiellement toxiques, il faut bien distinguer les déterminations relatives à l'ensemble des formes chimiques dans lesquelles le métal est impliqué (les " teneurs totales ») et celles relatives à seulement certaines formes (solubles, échangeables, associées à telle ou telle phase solide). Les teneurs totales incluent tous les ETM intégrés aux réseaux cristallins des minéraux primaires (ceux des roches) et secondaires (minéraux néoformés), notamment à ceux des silicates. Pour atteindre des teneurs vraiment " totales", il faut employer l'une des trois techniques analytiques suivantes : mise en solution par des acides fort dont l'acide fluorhydrique HF ; fusion alcaline ou fluorescence $\mathrm{X}$. Les teneurs obtenues après mise en solution à l'eau régale sont dites "pseudo-totales » car les silicates ne sont pas dissous en totalité.

\section{Notions De CPGN ET DE TAH}

La concentration pédo-géochimique naturelle (CPGN) est définie (Baize, 1997, chap. 1) comme la concentration d'un élément dans un horizon de sol, résultant uniquement de l'évolution géologique et pédologique, à l'exclusion de tout apport anthropique. D'un point à un autre d'un territoire, cette CPGN peut varier très largement, en fonction de la composition du matériau parental (héritage géochimique) et du type de sol qui s'y est développé (types de processus pédogénétiques et durée d'action de ces processus). Elle peut généralement être déterminée par l'étude des horizons pro- fonds des sols, le plus souvent restés indemnes des contaminations superficielles.

Tous les horizons de surface des sols cultivés ont reçu des apports d'éléments traces liés à des pratiques agricoles classiques (fertilisants, chaulages, fumiers, traitements phytosanitaires, apports d'oligo-éléments) ou à des épandages d'effluents ou de déchets (lisiers, composts, gadoues, boues de stations d'épuration). Ces horizons ont également reçu des retombées atmosphériques d'origine proches ou lointaines, d'origine naturelle (volcanisme) ou liées aux activités humaines, en quantités variables. En conséquence, une étude statistique des teneurs en ETM des horizons de surface labourés d'une série de sol ou d'un territoire ne peut pas permettre de déterminer la CPGN mais seulement une fourchette de valeurs "les plus fréquentes ", nommées «teneurs agricoles habituelles " (en abrégé TAH - Baize, 2002) (fig. 1), éventuellement « teneurs forestières habituelles".

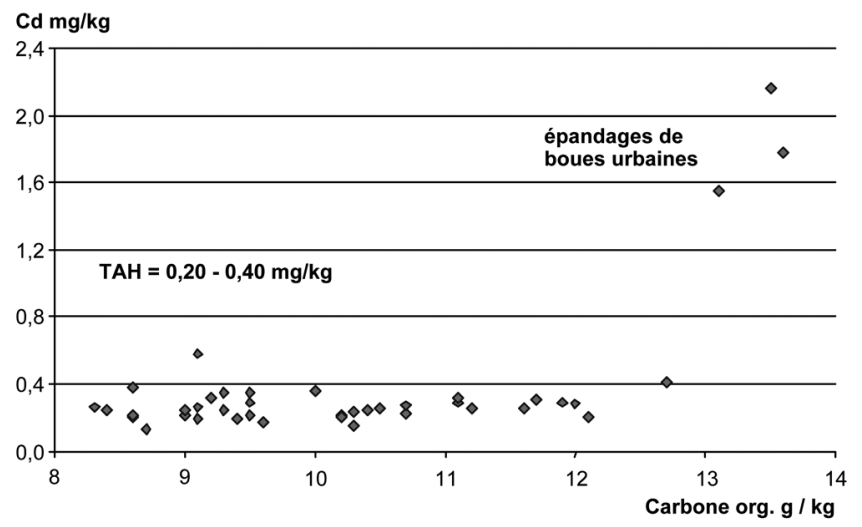

Figure 1 : Illustration de la notion de "teneur agricole habituelle» (TAH). Exemple de 39 horizons labourés de Néoluvisols issus de loess du Vexin français. On notera l'absence de relation entre les teneurs en cadmium et l'abondance du carbone organique. Les horizons de surface labourés de ce type de sol, quelle que soit leur teneur en carbone, présentent des teneurs agricoles habituelles en cadmium comprises entre 0,20 et $0,40 \mathrm{mg} / \mathrm{kg}$. Les trois valeurs beaucoup plus élevées correspondent à des contaminations par des épandages massifs de boues urbaines réalisées dans les années 70 . Figure 1: Illustration of the notion of usual agricultural content (UAC). The example of 39 ploughed horizons from Néoluvisols developed in loess (Vexin). There is no relationship between the cadmium contents and the abundance of organic carbon. The surface horizons of this soil type, whatever their carbon content, show cadmium UAC comprised between 0.20 and $0.40 \mathrm{mg} / \mathrm{kg}$. The three particular values much higher correspond to contaminations by heavy spreading of urban sewage sludge made during the seventies. 


\section{Teneurs totales dans les SOlS FRANÇais}

Des statistiques à l'échelle nationale ont été établies pour 11150 horizons de surface de sols agricoles (Baize et al., 2006). La médiane s'élève à $25,6 \mathrm{mg} / \mathrm{kg}$ soit un stock d'environ $100 \mathrm{~kg} / \mathrm{ha}$ (pour les 30 premiers centimètres). Le

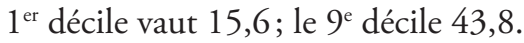

Le tableau 1 présente une comparaison entre les teneurs totales en plomb mesurées dans les horizons de surface et celles obtenues dans les horizons de profondeur prélevés sur les mêmes 260 sites, sous agriculture et sous forêts, en région Nord - Pas de Calais (Sterckeman et al., 2002). Tous les indicateurs statistiques sont nettement plus élevés dans les horizons de surface qui ont subi divers types d'apports liés à des activités humaines, anciennes ou récentes.

Dans l'horizon de surface des sols agricoles et forestiers, le plomb est donc pour une bonne part d'origine anthropique. Cet état de fait a débuté dès les premières activités minières des civilisations antiques bien avant la révolution industrielle et l'emploi des additifs plombés dans l'essence (Sungmin Hong et al., 1994).

Dans certains sols, développés dans des roches hautement minéralisées, les teneurs naturelles géochimiques sont beaucoup plus élevées, couramment comprises entre 80 et $400 \mathrm{mg}$ de $\mathrm{Pb}$ par kilogramme. De telles situations se retrouvent notamment sur tout le pourtour du Massif central, dans les formations sédimentaires en contact avec le socle.

\section{Mobilité DU PLOMB DANS LES SOLS}

Le plomb est réputé comme " très peu mobile " car fortement fixé par les matières organiques et/ou par les oxyhydroxydes de fer. Qu'il soit apporté par voie atmosphérique ou comme impureté indésirable dans divers épandages, le

\begin{tabular}{|l|c|c|}
\hline & \multicolumn{2}{|c|}{ Teneurs totales en plomb $(\mathrm{mg} / \mathrm{kg})$} \\
\hline \multicolumn{1}{|c|}{ nombre } & Surface & Non en surface \\
\hline Premier décile & 260 & 486 \\
\hline Médiane & 19,4 & 5,3 \\
\hline Neuvième décile & 29,3 & 16,2 \\
\hline Maximum & 51,3 & 24,9 \\
\hline
\end{tabular}

Tableau 1 : Référentiel Pédo-Géochimique du Nord-Pas de Calais. Statistiques comparées pour les horizons de surface et ceux non situés en surface (Sterckeman et al., 2002).

Table 1: Pedo-geochemical reference base for Nord-Pas de Calais. Comparison for some statistical indicators between surface soil horizons and deeper ones. plomb « exogène " a donc tendance à s'accumuler progressivement dans les horizons de surface des sols. Il s'avère un bon indicateur de la somme des apports d'origine humaine. Deux exemples sont présentés ici. Le tableau 2 montre l'exemple du site de l'Observatoire de la Qualité des Sols d'Evin-Malmaison situé seulement à $1 \mathrm{~km}$ de l'usine de traitement de métaux non ferreux Metaleurop (à Noyelles-godault). L'horizon de surface labouré (ici prélevé sur trois couches de $10 \mathrm{~cm}$ d'épaisseur chacune) est très chargé en plomb, tandis que l'horizon EB situé juste en dessous de la semelle de labour n'est que très peu affecté par la contamination. L'horizon BC situé entre 72 et $80 \mathrm{~cm}$ n'est pas contaminé.

La figure 2 présente les résultats obtenus pour des échantillons d'un même type de sol prélevés à différentes profondeurs à proximité d'usines traitant ou retraitant du plomb, en Beauce (Baize, 2002, chap. 21). Trois catégories d'horizons ont été distingués : les horizons de surface labourés (L), les horizons de moyenne profondeur et profonds ( $\mathrm{M}$ et $\mathrm{P}$ ) et les matériaux parentaux des sols, en l'occurrence des formations calcaires redistribuées par le vent au Quaternaire (FCR). Le diagramme a été volontairement limité sur sa droite à $80 \mathrm{mg} \mathrm{de} \mathrm{Pb} / \mathrm{kg}$. Les points surlignés en gris clair correspondent aux CPGN ou aux TAH locales de ce type de sol beauceron. Les valeurs surlignées en gris foncé correspondent à des contaminations par le plomb plus ou moins importantes. La plupart des horizons de surface ont été fortement contaminés par l'activité des usines (jusqu’à $550 \mathrm{mg} / \mathrm{kg}$ ) en fonction de la distance à la source, tandis que quelques horizons $M$ seulement ont été légèrement contaminés et présentent des teneurs en plomb comprises entre 30 et $40 \mathrm{mg} / \mathrm{kg}$ pour une CPGN de $29 \mathrm{mg} / \mathrm{kg}$.

\begin{tabular}{|c|c|c|}
\hline profondeur & horizon & $\mathrm{Pb}$ \\
\hline $\mathrm{cm}$ & & $\mathrm{mg} / \mathrm{kg}$ \\
\hline $0-12$ & $\mathrm{~L} 1$ & 500,0 \\
\hline $12-27$ & $\mathrm{~L} 2$ & 524,0 \\
\hline $27-34$ & $\mathrm{~L} 3$ & 525,0 \\
\hline $34-45$ & $\mathrm{~EB}$ & 33,4 \\
\hline $70-82$ & BC & 22,8 \\
\hline $106-124$ & $\mathrm{C} 2$ & 18,0 \\
\hline $142-175$ & C4 & 12,6 \\
\hline
\end{tabular}

Tableau 2 : Evin-Malmaison (à $1 \mathrm{~km}$ de la fonderie Metaleurop). Teneurs en plomb total en fonction de la profondeur. La forte contamination de l'horizon de surface (horizon L) n'a guère affecté les horizons situés sous la semelle de labour.

Table 2: Evin-Malmaison (a soil profile located at $1 \mathrm{~km}$ from the Metaleurop smelter). Total lead concentrations as a function of depth. The strong contamination of the topsoil hardly affected the deeper horizons under the plough pan. 


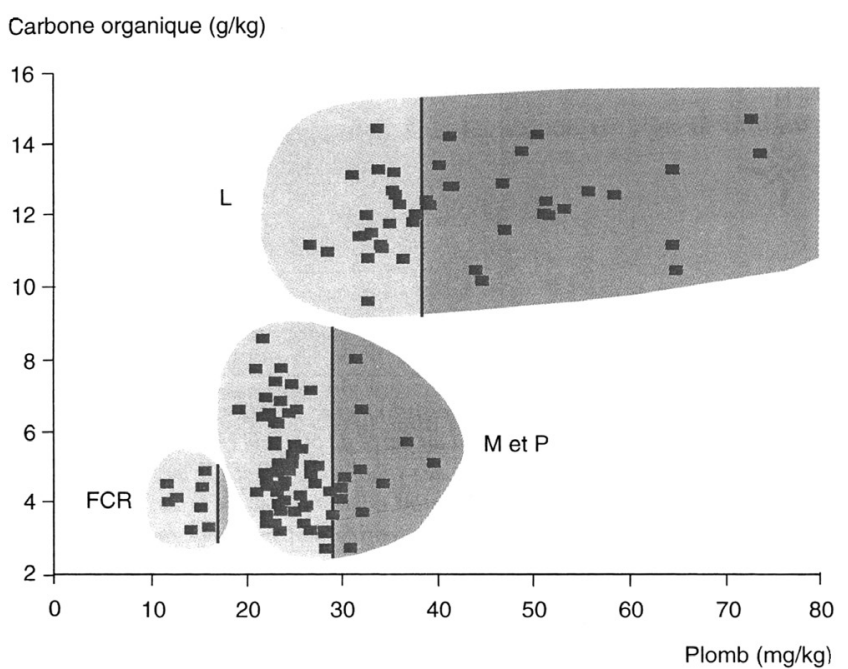

Figure 2 : Relation carbone organique/teneurs en plomb pour trois catégories d'horizons prélevés à proximité d'usines traitant du plomb (Baize, 2002). L = horizons de surface labourés. $M$ et $P$ $=$ horizons de moyenne profondeur $(40$ à $60 \mathrm{~cm})$ ou profonds $(>$ $70 \mathrm{~cm}) . \mathrm{FCR}=$ matériau parental calcaire.

Figure 2: Relationship between organic carbon and lead contents for three kinds of horizons sampled near two factories processing lead. $L$ = ploughed surface horizons. $M$ and $P=$ horizons at medium depth $(40$ to $60 \mathrm{~cm})$ or deeper $(>70 \mathrm{~cm})$. FCR = calcareous parent material.

Certains sols constituent cependant un cas particulier : les Podzosols où $\mathrm{Pb}$ (et d'autres métaux comme $\mathrm{Cu}$ ou $\mathrm{Zn}$ ) migrent verticalement dans le solum pour aller s'accumuler en profondeur (tableau 3) (Filipe, 1999; Semlali et al., 2000; Brännvall et al., 2001; Steinnes and Friedland, 2005). Un tel modèle pédogénétique extrême reste rare en France où les Podzosols ne se développent que dans des matériaux très acides et filtrants, dénués de phases capables de fixer fortement les métaux comme les minéraux argileux ou les oxy-hydroxydes de Fe et de Mn. Au contraire il y a formation de complexes organo-métalliques mobiles.
La possibilité inverse de remontées de $\mathrm{Pb}$ depuis la profondeur (absorption par les racines) vers la surface du sol (retombées des parties aériennes) par le cycle biogéochimique a été envisagée dans la littérature (Bruckert et al., 1979; Guillet et al., 1980; Denaeyer-DeSmet, 1980) mais elle reste très discutée en ce qui concerne le plomb (Heinrichs et Mayer, 1980). Même si les flux annuels sont très faibles, il faut envisager leur action cumulée sur de très longues périodes (plusieurs milliers d'années).

\section{FLUX DE RETOMBÉES ATMOSPHÉRIQUES ACTUELLES OU ANCIENNES}

Il y a peu de mesures disponibles, particulièrement en France. Les flux de plomb sont certainement très variables d'un point à un autre car on doit distinguer au moins trois types de retombées :

- les retombées diffuses planétaires qui affectent toute la surface du globe, même si les flux restent très faibles (Boutron, 1995);

- les retombées diffuses régionales : de nombreuses sources s'entremêlent, y compris des sources mobiles, l'impact s'étend sur des distances $<30 \mathrm{~km}$;

- les retombées locales à proximité d'une source bien identifiée, à une distance en général $<4 \mathrm{~km}$. C'est le cas des retombées péri-industrielles. Dans ce cas, on observe une décroissance rapide et exponentielle des teneurs totales mesurées en surface à mesure que l'on s'éloigne de la source, jusqu’à atteindre la TAH locale (Godin, 1985; Baize, 2002; Sterckeman et al., 2000) (cf. fig. 3 et 4).

Les chiffres trouvés dans la littérature internationale varient très largement de 1 à $85 \mathrm{~g} / \mathrm{ha} / \mathrm{an}$ pour les zones rurales éloignées de toute grande agglomération et de 100 à $400 \mathrm{~g} / \mathrm{ha} / \mathrm{an}$ en zones rurales de régions industrialisées de Pologne, d'Allemagne, de Wallonie ou d'Angleterre

\begin{tabular}{|c|c|c|c|c|c|c|c|}
\hline Horizon & Profondeur & Argile & Carbone organique & $\mathrm{pH}$ & $\mathrm{Pb}$ total & Pb stock & variation* \\
\hline & $\mathrm{cm}$ & $\%$ & $\%$ & & $\mathrm{mg} / \mathrm{kg}$ & $\mathrm{g} / \mathrm{m}^{3}$ & $\%$ \\
\hline litière & $+7-0$ & & 39,40 & & 25,5 & 0,15 & \\
\hline A & $0-45$ & 1,6 & 3,30 & 3,8 & 4,7 & 5,71 & -36 \\
\hline $\mathrm{E}$ & $45-100$ & 1,3 & 0,27 & 4,2 & 4,0 & 6,26 & -29 \\
\hline $\mathrm{BPh}$ & $100-155$ & 2,2 & 2,80 & 4,1 & 9,2 & 16,42 & +85 \\
\hline BPs & $155-210$ & 1,2 & 0,80 & 4,3 & 8,5 & 15,25 & +72 \\
\hline $\mathrm{C}$ & $>210$ & 0,7 & 1,20 & 4,1 & 5,7 & 8,87 & 0 \\
\hline
\end{tabular}

Tableau 3 : Podzosol issu de sables des Landes. Concentrations en plomb et bilans isovolumiques (Semlali et al., 2000).

Table 3: Podzosol developed in sandy material (Landes). Lead concentrations and isovolumetric balance. 


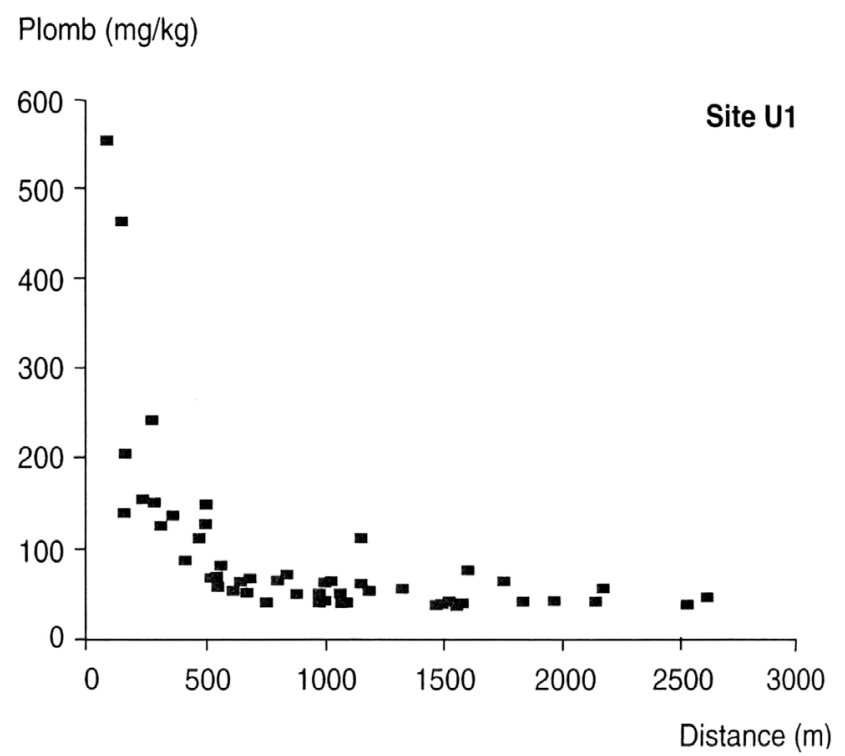

Figure 3 : Concentrations en plomb dans les horizons de surface en fonction de la distance à l'usine la plus proche (Beauce) (Baize, 2002).

Figure 3: Lead concentrations in surface horizons as a function of the distance to the nearest factory (Beauce).

(Galloway et al., 1982; Navarre et al., 1980; Alloway et al., 1999).

Quelques rares études fines françaises sur l'évolution des teneurs en plomb dans les sols ont fourni des chiffres plus fiables : $930 \mathrm{~g} / \mathrm{ha} / \mathrm{an}$ à Versailles entre 1929 et 1984 (Juste et Tauzin, 1986); $650 \mathrm{~g} / \mathrm{ha} / \mathrm{an}$ entre 1938 et 1999 à Grignon (Yvelines) (Baize et Bourgeois, 2005); 50 à $300 \mathrm{~g} / \mathrm{ha} / \mathrm{an}$ au cours de la période 1990-2000 à Versailles (Semlali et al., 2004).

Outre leur grande variabilité dans l'espace, les flux de retombées atmosphériques ont certainement varié largement au cours du temps. Depuis l'Antiquité, plusieurs périodes d'activités minières et industrielles ont alterné avec des périodes d'arrêt de ces activités et toutes ces variations sont susceptibles d'être enregistrées dans des sédiments ou des tourbières (Sugden et al., 1991; Steinnes, 1997; Monna et al., 2004; Jensen, 2004). Au cours des temps modernes, une forte croissance des retombées atmosphériques de plomb a correspondu avec l'aube de la révolution industrielle puis une décroissance nette est intervenue avec l'abandon de l'essence plombée (e.g. Miller et Friedland, 1994; Candelone and Sungmin Hong, 1995; Azimi et al., 2003).

Ainsi, les travaux de Shotyk menés sur des tourbières suisses ont montré que des activités minières et industrielles antiques avaient été enregistrées et pouvaient être encore reconnues aujourd'hui, plusieurs milliers d'années plus tard.

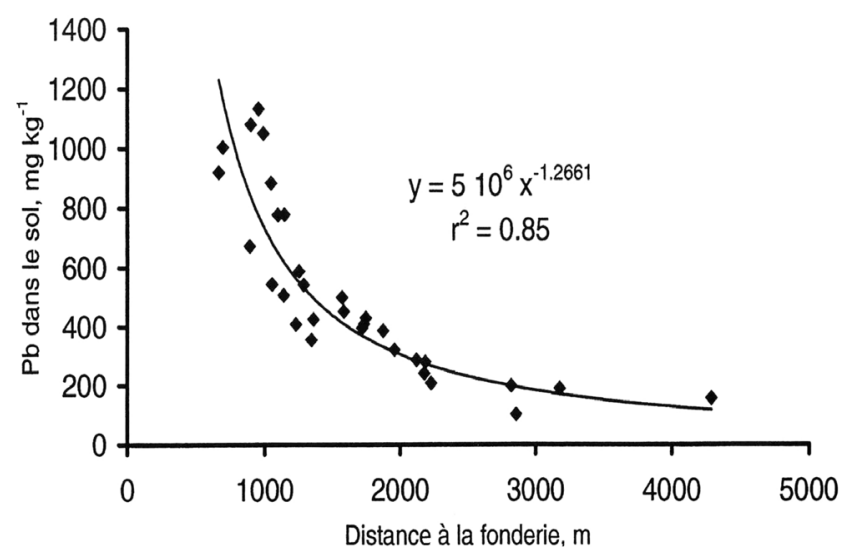

Figure 4 : Concentrations en plomb dans les horizons de surface des sols en fonction de la distance à la fonderie de NoyellesGodault (Sterckeman et al., 2000).

Figure 4: Lead concentrations in surface horizons as a function of the distance to the smelter at Noyelles-Godault.

Ces activités étaient localisées fort loin de Suisse et les flux enregistrés ont grandement varié dans le temps (Shotyk and Blaser, 1999; Shotyk et al., 1998 et 2000).

\section{COMMENT DÉCELER UNE CONTAMINATION ANTHROPIQUE ET ÉVALUER SON NIVEAU?}

La méthode la plus simple consiste à comparer la teneur totale mesurée en surface et les concentrations totales mesurées en profondeur. Mais il faut prendre également en compte les épaisseurs des horizons et leur densité apparente. Il est donc préférable de raisonner en stocks.

En effet, sous forêt, le plomb est susceptible de s'accumuler sur les 4 à 10 premiers centimètres très humifères et les teneurs deviennent souvent très faibles ensuite, correspondant aux CPGN locales. La concentration mesurée dans le même type de sol cultivé et situé à proximité peut souvent sembler bien inférieure, mais le stock calculé sur les 30 premiers $\mathrm{cm}$ est le même (tableau 4).

\section{RTE : CONCEPT ET UTILISATION}

Colbourn et Thornton (1978) ont travaillé sur les sols d'un secteur minier près de Sheffield et, reprenant les travaux d'autres auteurs britanniques, ont proposé d'utiliser le "relative topsoil enhancement" (RTE) comme indicateur de contamination anthropique. Le RTE est défini comme le rapport de la teneur en $\mathrm{Pb}$ dans la tranche $0-15 \mathrm{~cm} / \mathrm{la}$ teneur 


\begin{tabular}{|c|c|c|c|c|}
\hline & \multicolumn{2}{|c|}{$\mathrm{Pb}$ concentrations $\mathrm{mg} / \mathrm{kg}$} & \multicolumn{2}{c|}{$\mathrm{Pb}$ stocks $\left(\mathrm{en} / \mathrm{m}^{2}\right)$} \\
\hline & forêt & culture & forêt & culture \\
\hline $0-10 \mathrm{~cm}$ & 86 & 29,5 & 7,74 & 3,84 \\
\hline $10-20 \mathrm{~cm}$ & 18 & 29,5 & 1,98 & 3,84 \\
\hline $20-30 \mathrm{~cm}$ & 16 & 29,5 & 1,92 & 3,84 \\
\hline & & total & 11,64 & 11,52 \\
\hline
\end{tabular}

Tableau 4: Comparaison des concentrations et des stocks de plomb entre un sol forestier et le même sol labouré régulièrement. Calcul sur les 30 premiers centimètres.

Table 4: Comparison of lead concentrations and stocks between a forested soil and the same soil frequently ploughed. Calculation for the 30 upper centimetres.

en $\mathrm{Pb}$ dans la tranche de sol située entre 30 et $45 \mathrm{~cm}$ de profondeur. Les sols des secteurs contaminés par les activités minières et les fonderies montraient des valeurs du RTE comprises entre 4 et 20 tandis que les sols éloignés de ces sources contaminantes présentaient des valeurs de l'ordre de 1,2 à 2,0 seulement.

La démarche de calcul du RTE est fondée sur l'hypothèse de la non migration en profondeur du plomb anthropogène. Le plomb, nous l'avons déjà évoqué, est reconnu comme un métal particulièrement peu mobile mais il y a des exceptions. Elle semble également fondée implicitement sur l'idée qu'une répartition naturelle du plomb (i.e. sans intervention des activités humaines) devrait mener à un RTE proche de 1,0 . Or nous savons que toutes les « entrées " d'ETM dans les sols se font "par leur surface " qu'il s'agisse des flux anthropiques (retombées atmosphériques, apports agricoles, épandages) ou des flux liés au cycle biogéochimique via les parties aériennes des plantes.

Une étude (non publiée) a été menée sur le calcul des RTE dans plusieurs bases de données (programme ASPITET) (Baize, 1997; RPG-NPC Sterckeman et al., 2002). En voici les principales conclusions. Le RTE est un indice arithmétique dont l'interprétation doit être modulée en fonction de considérations numériques et géo-pédologiques.

Numériques: $32 / 8$ ne peut pas être interprété de la même façon que 108/27 et pourtant le RTE correspondant est de 4,0 dans les deux cas.

Géo-pédologiques : pour interpréter correctement un RTE il faut connaître la nature et l'origine du matériau parental et prendre en considération des critères fins comme le type d'horizon servant de référence en profondeur, et certaines caractéristiques analytique comme sa teneur en calcaire.

On observe des RTE assez élevées (en absence de contamination nette) dès que l'on mesure une valeur très faible au dénominateur, donc dans l'horizon situé plus en profondeur. C'est le cas des sols développés dans des matériaux géolo- giques très pauvres en $\mathrm{Pb}$ (sables quartzeux, amphibolites, basaltes) ou dans des matériaux hyper-calcaires, également très pauvres en $\mathrm{Pb}$ (craies et matériaux crayeux).

On observe des RTE inférieures à 1,0 lorsque l'horizon de profondeur est nettement plus argileux que l'horizon de surface (cas des Luvisols et des Planosols ainsi que des sols modérément appauvris en argile en surface) ou bien lorsque les horizons profonds se sont développés à partir de roches minéralisées par des processus géologiques naturels (tableau 5; fig. 7).

La nature de l'horizon profond prélevé (granulométrie, taux de calcaire, accumulation de telle ou telle substance) peut jouer grandement sur la valeur numérique du RTE, et ce à quelques centimètres près (par exemple prélèvement et analyse d'un horizon illuvial BT au lieu d'un horizon éluvial E).

A moins de parfaitement connaître les valeurs habituelles de telle ou telle série de sols (et donc de connaître avec précision les valeurs normales de RTE pour cette série), il n'est pas possible d'interpréter avec certitude comme notoirement contaminés des solums présentant une RTE inférieure à 6 .

\section{DémARCHE DE COMPARAISON VERTICALE}

Une approche par comparaison verticale est donc préférable. Cela consiste à établir le profil des teneurs en plomb en fonction de la profondeur, ce qui implique de faire réaliser 3-4 ou 5 mesures superposées dans différents horizons (fig. 5).

Lorsque la teneur est très forte en surface, puis diminue rapidement avec la profondeur, il s'agit très probablement d'une contamination anthropique. La fig. 6 fournit un exemple, ainsi que le tableau 2. Lorsque, au contraire, la teneur est forte en surface et continue de croître avec la pro-

\begin{tabular}{|c|c|c|c|c|}
\hline $\begin{array}{c}\text { profond. } \\
\mathrm{cm}\end{array}$ & horizon & $\begin{array}{c}\text { argile } \\
\%\end{array}$ & $\begin{array}{c}\mathrm{Pb} \\
\mathrm{mg} / \mathrm{kg}\end{array}$ & RTE \\
\hline $0-26$ & LE & 24,7 & 134 & \\
\hline $26-40$ & BT & 53,1 & 192 & 0,70 \\
\hline $40-58$ & BT & 52,4 & 218 & \\
\hline $58-90$ & BT & 59,2 & 245 & \\
\hline
\end{tabular}

Tableau 5 : Distribution verticale des teneurs en plomb dans le solum Vault-de-Lugny (Avallonnais). Forte anomalie géochimique naturelle (calcaire sinémurien minéralisé).

Table 5: Vertical distribution of lead concentrations in the soil profile "Vault-de-Lugny" (near Avallon). Strong natural anomaly. The underlying Sinemurian limestone is strongly mineralized. RTE = "relative topsoil enhancement». 
PRINCIPE de la

\section{COMPARAISON VERTICALE}

TENEUR EN ÉLÉMENT TRACE

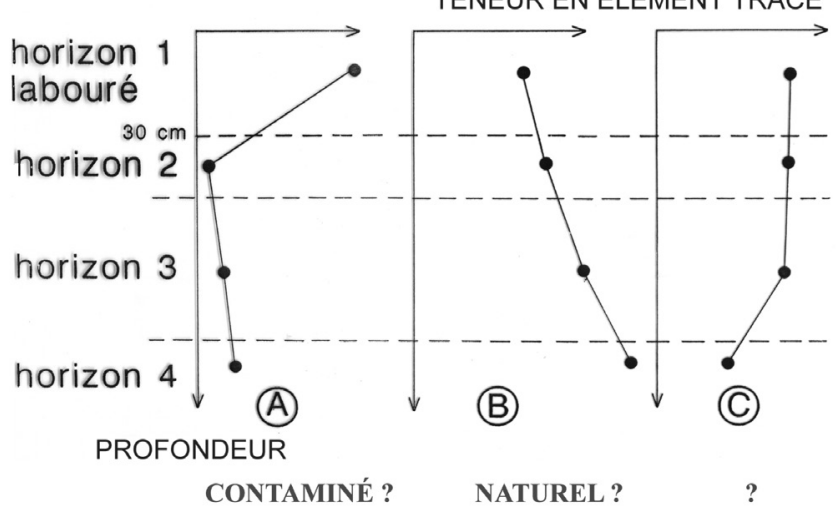

Figure 5 : Principe de la comparaison verticale (Baize, 1997). Figure 5: The vertical comparison. Principle.

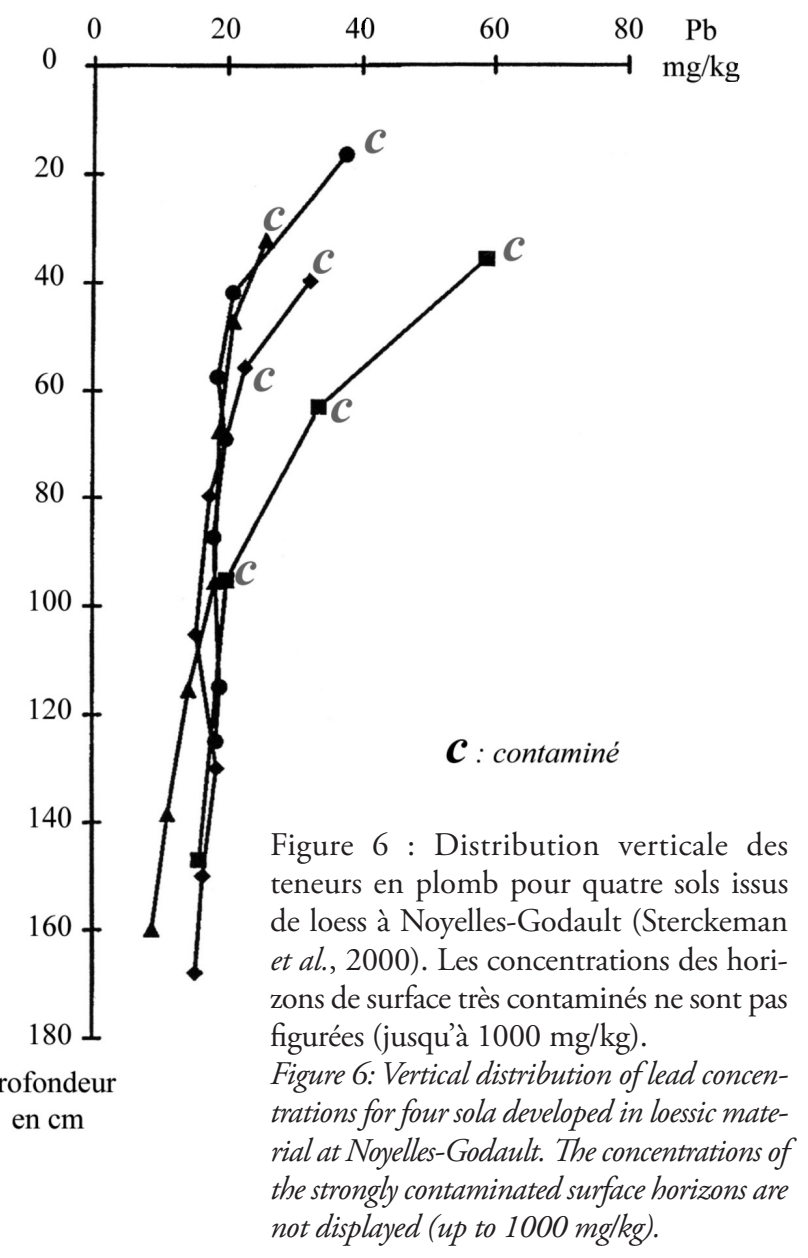

a

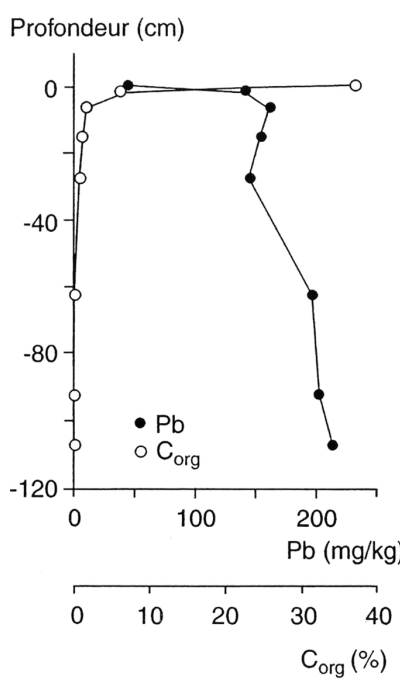

Figure 7 : Solum du bois de Belle Verne (Morvan nord) (Lévêque et al., 2002). a) Distribution verticale du carbone organique et du plomb. b) Distribution verticale du rapport isotopique ${ }^{206} \mathrm{~Pb} /{ }^{207} \mathrm{~Pb}$. Figure 7: Soil profile "Bois de Belle Verne" (northern Morvan). a) Vertical distribution of organic carbon and lead. b) Vertical distribution of the isotopic ratio ${ }^{206} \mathrm{~Pb} /{ }^{207} \mathrm{~Pb}$.

fondeur, il s'agit plutôt d'une anomalie naturelle (tableau 5 et fig. 7).

Lorsque le profil du plomb est relativement uniforme avec des valeurs élevées, puis avec une forte diminution en profondeur, le doute subsiste. Cette diminution peut impliquer une forte contamination du solum ne se limitant pas à l'horizon de surface mais elle peut résulter également de l'existence en profondeur d'un horizon ou d'un matériau très calcaire, le carbonate de calcium jouant alors le rôle d'un diluant vis-à-vis de la plupart des ETM (Baize, 1997).

D'autres techniques sont alors nécessaires pour trancher, notamment les techniques isotopiques (Semlali et al., 2000 et 2004; Lévêque et al., 2002; Cloquet et al., 2006 , parmi bien d'autres).

Cette démarche de " comparaison verticale " et de prise en compte des CPGN et TAH locales sont des approches à la fois très simples et faciles à mettre en œuvre à un coût relativement modéré.

\section{Cas Particulier des ZONES Minières}

Les mines de plomb ont été implantées évidemment là où les roches (et donc les sols naturels qui en sont issus par altération) étaient riches en plomb. D’où la difficulté 
aujourd'hui de distinguer le plomb naturellement présent dans le sol (CPGN souvent élevée) et le plomb anthropique lié aux activités minières (extraction, traitements, haldes). D'autant qu'ils ont souvent la même signature isotopique!

\section{Bibliographie}

Alloway, B. J., Zhang, P., Mott, C. J. B., Nicholson, F., Chambers, B., Smith, S., Carlton-Smith, C., 1999. Atmospheric deposition of heavy metals onto agricultural land in England and Wales, Proc. 5th Intern., Conf. on the Biogeochemistry of Trace Elements, Wien, p. 414-415.

Azimi, S., Ludwig, A., Thévenot, D. R., Colin, J.-L., 2003. Trace metal determination in total atmospheric deposition in rural and urban areas, Science of the Total Environment, 308, p. 247-254.

BAIzE D., 1997. Teneurs totales en éléments traces métalliques dans les sols (France). Références et stratégies d'interprétation, Paris, INRA Éditions, $410 \mathrm{p}$.

BaIze D., 2002. Pollutions par le plomb. Impact de trois établissement industriels sur les sols cultivés environnants, in Baize, D. et Tercé, M. (coord.), Les Éléments traces métalliques dans les sols - Approches fonctionnelles et spatiales, Paris, INRA Éditions, p. 351-374.

Baize, D., Bourgeois, S., 2005. Estimation des apports agricoles et des retombées atmosphériques en éléments en traces et majeurs grâce à un essai de longue durée (dispositif Dehérain à Grignon), Étude et Gestion des Sols, 12, 1, p. 9-23.

Baize, D., Saby N., Deslais W, Bispo A., Feix I., 2006. Analyses totales et pseudo-totales d'éléments en traces dans les sols Principaux résultats et enseignements d'une collecte nationale, Etude et Gestion des Sols, 2, p. 181-200.

Boutron, C. F., 1995. Historical reconstruction of the earth's past atmospheric environment from Greenland and Antarctic snow and ice cores, Environmental Reviews, vol. 3, p. 1-28.

Brännyall, M.-L., Bindler, R., Emteryd, O., Renberg, I., 2001. Vertical distribution of atmospheric pollution lead in Swedish boreal forest soils, Water Air Soil Pollution Focus 1 (3/4), p. 357-370.

Bruckert, S., Cachau-Herreillat, F., Capdecomme, H., GuILleT, B., 1979. Intervention du cycle biogéochimique dans la genèse des concentrations anomaliques du plomb. Exemple de la vallée du Rahin (Haute Saône, France), Bull. Cent. Rech. Exploration-Production Elf-Aquitaine, 3, 1, p. 281-297.

Candelone, J.-P., Sungmin, H., 1995. Post-Industrial Revolution changes in large-scale atmospheric pollution of the northern hemisphere by heavy metals as documented in central Greenland snow and ice, Journ. of Geophysical Research, vol 100, D8, p. 16.605-16.616.
Cloquet, C., Carignan, J., Libourel, G., Sterckeman, T., Perdrix, E., 2006. Tracing source pollution in soils using cadmium and lead isotopes, Environmental Science \& Technology, 40 (8), p. 2525-2530.

Colbourn P., Thornton I., 1978. Lead pollution in agricultural soils. Journal of Soil Science, 29, p. 513-526.

Denaeyer-De Smet S., 1974. Cycle biologique annuel et distribution du plomb dans une pessière et une hêtraie établies sur même roche-mère, Bull. Soc. Roy. Bot. Belge, 107, p. 115-125.

FILIPE, D., 1999. Étude de la localisation et essai de spéciation des métaux $\mathrm{Pb}, \mathrm{Zn}, \mathrm{Cu}, \mathrm{Cd}$ dans un sol podzolique sous forêt acide, Mémoire de DAA - ENSA Rennes, 64 p.

Galloway, J. N., Thornton, J. D., Norton, S. A., Volchok, H. L., McLean, R. A. N., 1982. Trace metals in atmospheric deposition : a review and assessment, Atmospheric Environment, vol. 16, $\mathrm{n}^{\circ}$ 7, p. 1677-1700.

Godin, P. M., Feinberg, M. H., Ducauze, C. J., 1985. Modelling of soil contamination by airborne lead and cadmium around several emission sources, Environmental Pollution (series B), 10, p. 97-114.

Guillet, B., Jeanroy, E., Rougier, C., Souchier, B., 1980. Le cycle biogéochimique et la dynamique du comportement des éléments-traces $(\mathrm{Cu}, \mathrm{Pb}, \mathrm{Zn}, \mathrm{Ni}, \mathrm{Co}, \mathrm{Cr}$ ) dans les pédogenèses organiques acides. L'exemple des sols brunifiés et podzoliques sur le granite des Ballons, Vosges méridionales, Centre de Pédologie Biologique CNRS, Nancy, note technique $\mathrm{n}^{\circ} 27$, $58 \mathrm{p}$.

Heinrichs, H., MaYer, R., 1980. The role of forest vegetation in the biogeochemical cycle of heavy metals, Jour. Environ. Qual., Vol. 9, n 1, p. 111-118.

Jensen, A., 1997. Historical deposition rates of $\mathrm{Cd}, \mathrm{Cu}, \mathrm{Pb}$, and $\mathrm{Zn}$ in Norway and Sweden estimated by $210 \mathrm{~Pb}$ dating and measurement of trace elements in cores of peat bogs, Water, Air, \& Soil Pollution, 95, 1-4, p. 205-220.

Juste, C., TAuzin, J., 1986. Évolution du contenu en métaux lourds d'un sol de limon maintenu en jachère nue après 56 années d'application continue de divers engrais et amendements, C.R. Acad. Agriculture Fr., 72, 9, p. 739-746.

Lévêque, J., Philippe, S., Baize, D., Monna, F., Andreux, F., HaACK, U., 2002. Pollutions par le plomb. Utilisation des isotopes stables pour la détermination de l'origine (géogène ou industrielle) du plomb, in BAIze, D. et Tercé, M. (coord.), Les Éléments traces métalliques dans les sols - Approches fonctionnelles et spatiales, INRA Éditions, Paris, p. 375-391.

Miller, E. K., Friedland, A. J., 1994. Lead migration in forest soils : response to changing atmospheric inputs, Environmental Science \& Technology, 28, 4, p. 662-669.

Monna, F., Petit, C., Gulllaumet, J.-P., Jouffroy-Bapicot, I., Blanchot, C., Dominik, J., Losno, R., Richard, H., LÉvêque, J., Château, C., 2004. History and environmental 
impact of mining activity in Celtic Aeduan territory recorded in a peat bog (Morvan, France), Environmental Science \& Technology, 38, 3, p. 665-673.

Navarre, J.-L., Ronneau, C., Priest, P., 1980. Deposition of heavy elements on belgian agricultural soils, Water, Air and Soil Pollution, 14, p. 207-213.

Semlali, R.-M., vanOort, F., Loubet, M., Denaix, L., 2000. La composition isotopique du plomb : un outil privilégié pour l'estimation de la distribution du plomb anthropique et naturel dans les sols, C.R. Acad. Sciences, Paris, 331, p. 595-600.

Semlali, R., Dessogne, J.-B., Monna, F., Bolte, J., Azimi, S., Navarro, N., Denaix, L., Loubet, M., Château, C., Van Oort, F., 2004. Modeling lead input and output in soils using lead isotopic geochemistry, Environmental Science \& Technology, 38, (5), p. 1513-1521.

Shotyk, W., Weiss, D., Appleby, P. G., Cheburkin, A. K., Frei, R., Gloor, M., Kramers, J. D., Reese, S., Van der Knap, W. O., 1998. History of atmospheric lead deposition since 12,370 14C yr BP from a peat bog, Jura Mountains, Switzerland, Science, vol. 281, p. 1635-1640.

SHOtyK, W., Blaser, P., 1999. Independant records of long-term atmospheric $\mathrm{Pb}$ deposition to Swiss forest soils using peat cores from ombotrophic bogs, 5th Intern. Conf. on the Biogeoch. of Trace Elements, Wien, p. 1076-1077.

Shotyk, W., Blaser, P., Grunig, A., Cheburkin, A. K., 2000. A new approach for quantifying cumulative, anthropogenic atmospheric lead deposition using peat cores from bogs:
$\mathrm{Pb}$ in eight Swiss peat bogs profiles, The Science of the Total Environment, 249, p. 281-295.

STEINNES, E., 1997. Trace element profiles in ombrogenous peat cores from Norway: evidence of long range atmospheric transport, Water, Air, \& Soil Pollution, 100, 3-4, p. 405-413.

Steinnes, E., Friedland, A. J., 2005. Lead migration in podzolic soils from Scandinavia and the United States of America, Can. J. Soil Science, 85, p. 291-294.

Sterckeman, T., Douay, F., Proix, N., Fourrier, H., 2000. Vertical distribution of $\mathrm{Cd}, \mathrm{Pb}$ and $\mathrm{Zn}$ in soils near smelters in the North of France, Environmental Pollution, 107 (3), p. 377-389.

Sterckeman, T., Douay, F., Fourrier, H., Proix, N. Référentiel Pédo-Géochimique du Nord-Pas de Calais. Rapport final (130 p.) et Annexes (306 p) [http://www.lille.inra.fr/Documents/ rpg.htm].

Sugden, C. L., Farmer, J. G., Mackenzie, A. B., 1991. Lead and $206 \mathrm{~Pb} / 207 \mathrm{~Pb}$ profiles in $210 \mathrm{~Pb}$-dated ombrotrophic peat cores from Scotland, Intern. Conf. Heavy Metals in the Environment, Edimburgh, Vol. 1, p. 90-93.

Sungmin, H., Candelone, J. P., Patterson, C. C., Boutron, C. F., 1994. Greenland ice evidence of hemispheric lead pollution two millenia ago by Greek and Roman civilizations, Science, vol. 265, p. 1841-1843.

[http:/letm.orleans.inra.fr/index.html]. 
